\title{
Correlation of p16 and nm23-H1 expression levels with incidence and prognosis of soft tissue sarcoma
}

\author{
JINPENG JIA ${ }^{1}$, PEI YIN ${ }^{2}$, XINGPENG ZHANG $^{1}$, FEI YANG ${ }^{3,4}$, GE SONG $^{1}$, \\ WENZHI BI ${ }^{1}$, GANG HAN ${ }^{1}$, MENG XU ${ }^{1}$, WEI WANG ${ }^{1}$ and XIAOQUE ZHENG ${ }^{1}$ \\ ${ }^{1}$ Department of Orthopaedics, General Hospital of Chinese People's Liberation Army, Beijing 100853; \\ ${ }^{2}$ Department of Nephrology, First Affiliated Hospital of Chinese PLA General Hospital, Beijing 100037; \\ ${ }^{3}$ Beijing National Laboratory for Molecular Sciences State Key Laboratory of Polymer Physics \\ and Chemistry Institute of Chemistry Chinese Academy of Sciences, Beijing 100190; \\ ${ }^{4}$ University of Chinese Academy of Sciences, Shijingshan, Beijing 100049, P.R. China
}

Received May 9, 2018; Accepted February 18, 2019

DOI: $10.3892 / \mathrm{ol} .2019 .10145$

\begin{abstract}
The expression levels of p16 and nm23-H1 genes in soft tissue sarcoma (STS) were evaluated to investigate correlation of the expression levels with the incidence and prognosis of STS. Tumor tissues and para-carcinoma normal tissues were collected from 64 STS patients. The messenger ribonucleic acid (mRNA) expression levels of p16 and nm23-H1 in the tissues were detected via reverse transcription-quantitative polymerase chain reaction (RT-qPCR), and the protein expression levels of p16 and nm23-H1 in tissues were detected using immunohistochemistry. Spearman's correlation analysis was used for the correlation between expression levels of p16 and $\mathrm{nm} 23-\mathrm{H} 1$ in STS tissues and the correlation between p16 and $\mathrm{nm} 23-\mathrm{H} 1 \mathrm{mRNA}$ and protein expression. Moreover, the correlation of p16 and $\mathrm{nm} 23-\mathrm{H} 1$ expression levels in tumor tissues with pathological parameters and prognosis of STS patients were analyzed combined with clinical data. Results of RT-qPCR showed that mRNA expression levels of p16 and $\mathrm{nm} 23-\mathrm{H} 1$ in tumor tissues of STS patients were significantly lower than those in para-carcinoma normal tissues $(\mathrm{P}<0.01)$. Results of immunohistochemistry showed that the positive expression rates of p16 and $\mathrm{nm} 23-\mathrm{H} 1$ in tumor tissues of STS patients (43.75 and 39.06\% respectively) were significantly lower than those in para-carcinoma normal tissues (85.93 and $89.06 \%$ respectively). The expression of p16 and nm23-H1 mRNA was positively correlated with protein expression levels. There was a positive correlation between the expression levels of p16 and nm23-H1 in tumor tissues of STS patients. The negative expression of p16 in tumor tissues of STS patients
\end{abstract}

Correspondence to: Dr Fei Yang, Beijing National Laboratory for Molecular Sciences State Key Laboratory of Polymer Physics and Chemistry Institute of Chemistry Chinese Academy of Sciences, Zhongguancun North First Street 2, Beijing 100190, P.R. China E-mail: feiqin25974@163.com

Key words: STS, p16, nm23-H1, prognosis correlated with tumor size, tumor metastasis and clinical staging, and the negative expression of nm23-H1 correlated with tumor metastasis and clinical staging. The overall 5-year survival rate of patients was $54.68 \%$, and the prognosis of patients with positive expression levels of p16 and nm23-H1 was better. Univariate survival analyses revealed that p16 and $\mathrm{nm} 23-\mathrm{H} 1$ were influencing factors of the overall survival rate of STS patients. p16 and nm23-H1 expression in STS is low, and their expression levels are closely related to the pathological parameters and prognosis of STS patients, so they can serve as reference indexes for prognosis estimation of STS.

\section{Introduction}

Soft tissue sarcoma (STS) is a malignant tumor derived from mesenchymal tissues. The worldwide prevalence rate of STS is as high as 1.8-5.0/100,000 every year, and it accounts for only $1 \%$ in malignant tumors of adults, but as high as $10 \%$ in children's tumors. Currently, more than 50 kinds of STS subtypes have been definitely diagnosed clinically $(1,2)$. There are approximately 11,000 new cases of STS each year in the United States (3), but as many as 2.0/100,000 new cases each year in China (4). In all STS, pleomorphic sarcoma, also known as malignant fibrous histiotoma in clinic, has the highest incidence rate, accounting for $25-35 \%$, followed by liposarcoma (25\%), and the incidence rates of malignant schwannoma and leiomyosarcoma are relatively lower. The incidence rate of STS in males is higher than that in females, and it mainly occurs in the age group of 30-50 years and in any part of the body, mainly the trunk, head and neck, and limbs (5). STS has not only complex typing, but also high heterogeneous degree. Moreover, the growth of STS is characterized by destruction and infiltration, and its prognosis is poor and postoperative recurrence occurs easily because of its strong invasion (6).

p16 protein, a kind of tumor suppressor protein, can bind to cyclin-dependent kinase 4 (CDK4), and its function of preventing the normal cell cycle is exerted by inhibiting $\mathrm{pRB}$ protein phosphorylation and blocking CDK4 complex formation (7). nm23 gene is a key gene involved in the invasion of malignant tumors, and was isolated by Steeg et al (8) in the 
complementary deoxyribonucleic acid (cDNA) library with mouse melanoma K-1735 cell lines as the research objects. Studies have shown that the nm23 gene plays inhibitory and regulatory roles in the outcome of cancer cells, significantly inhibiting the proliferation and invasion of tumor cells (9). In addition, abnormal changes in the nm23 gene widely exist in malignant tumors, which are closely related to cancer cell invasion (10).

The occurrence and development of osteosarcoma is a process of gradual evolution, which eventually develops into a highly invasive tumor. The existence of periodic dysfunction and changes in the expression of invasion-associated genes are worthy of full study. Therefore, we selected to study the expression of p16 and $\mathrm{nm} 23-\mathrm{H} 1$ and its relationship with the pathogenesis and prognosis of soft tissue sarcoma. In order to investigate the expression levels of p16 and nm23-H1 in STS and the correlations of their expression levels with the incidence and prognosis of STS, messenger ribonucleic acid (mRNA) and protein expression levels of p16 and nm23-H1 in STS tissues were detected via reverse transcription-quantitative polymerase chain reaction (RT-qPCR) and immunohistochemical method, respectively, in this study, and the relationship between the expression levels of p16 and nm23-H1 was analyzed.

\section{Patients and methods}

Materials. Frozen and paraffin specimens of tumor tissues and corresponding para-carcinoma normal tissues were collected from 64 STS patients treated in the General Hospital of Chinese People's Liberation Army (Beijing, China), from January 2007 to January 2012. Patients were aged 11-72 years, including 35 males and 29 females, all of them were pathologically diagnosed with STS, and they had complete clinical data and received surgical treatment in the hospital.

This study was reviewed and approved by the Clinical Ethics Committee of General Hospital of Chinese People's Liberation Army, and all patients or their families signed an informed consent.

TRIzol RNA extraction kit (Takara, Dalian, China), primer synthesis, RT kits and quantitative SYBR-Green PCR kits (Ambion; Thermo Fisher Scientific, Inc., Waltham, MA, USA), primary rabbit anti-human p16 and nm23-H1 antibodies (1:100; cat. nos. 10883-1-AP and 11086-2-AP, respectively; Proteintech Group, Inc., Wuhan, China), immunohistochemical staining kit SP-9001 (Beijing Zhongshan Goldenbridge Biotechnology Co., Ltd., Beijing, China).

$R T$ - $q P C R$. Approximately $50 \mathrm{mg}$ tumor tissues and para-carcinoma normal tissues cryopreserved in liquid nitrogen were used, and the total RNA was extracted from specimens using the TRIzol kit. Qualified RNA specimens [absorbance (A) ${ }_{260} /$ $\mathrm{A}_{280}$ ratio of 1.8-2.0] were selected for reverse transcription to obtain cDNA. PCR was carried out with cDNA as a template according to the manufacturer's instructions. The specific reaction conditions were as follows: pre-denaturation at $94^{\circ} \mathrm{C}$ for $4 \mathrm{~min}$, denaturation at $94^{\circ} \mathrm{C}$ for $1 \mathrm{~min}$, annealing at $50^{\circ} \mathrm{C}$ for $50 \mathrm{sec}$, extension at $72^{\circ} \mathrm{C}$ for $1 \mathrm{~min}$, amplification for 35 cycles, and extension for $10 \mathrm{~min}$. Glyceraldehyde-3-phosphate dehydrogenase (GAPDH) mRNA was used as a control, the primer
Table I. RT-qPCR primer sequences.

\begin{tabular}{lc}
\hline Genes & \multicolumn{1}{c}{ Primer sequences } \\
\hline p16 & F: 5'-GAAGAAAGAGGAGGGGCTG-3' \\
& R: 5'-GCGCTACCTGATTCCAATTC-3' \\
nm23-H1 & F: 5'-GTGAAAAGCAATGTGGT-3' \\
& R: 5'-TTGCCATGGTCTGGGAG-3' \\
GAPDH & F: 5'-ATGGCACCGTCAAGGCTGAG-3' \\
& R: 5'-GCAGTGATGGCATGGACTGT-3' \\
\hline
\end{tabular}

F, forward; R, reverse; RT-qPCR, reverse transcription-quantitative polymerase chain reaction.

Table II. Correlation between p16 and nm23-H1 protein expression levels in STS tissues.

\begin{tabular}{lrrrr}
\hline & \multicolumn{2}{c}{$\mathrm{nm} 23-\mathrm{H} 1$} & & \\
\cline { 2 - 3 } p16 & Positive & Negative & r value & P-value \\
\hline Positive & 20 & 8 & 0.722 & $<0.01$ \\
Negative & 5 & 31 & & \\
\hline
\end{tabular}

sequences are shown in Table I, and the experimental results were analyzed using the $2^{-\Delta \mathrm{Cq}}$ method (11).

Immunohistochemistry. Streptavidin peroxidase (SP) method was used in immunohistochemical assay in accordance with instructions of the SP-9001 kit. The tissue was fixed with $40 \%$ formaldehyde at $20^{\circ} \mathrm{C}$ for $12 \mathrm{~h}$. The paraffin sections were dewaxed and inactivated with $3 \% \mathrm{H}_{2} \mathrm{O}_{2}$, followed by antigen retrieval via microwave twice $\left(750 \mathrm{~W}, 95^{\circ} \mathrm{C}\right.$, once every $5 \mathrm{~min}$ ). A total of $5 \mu \mathrm{l}$ sections was prepared and blocked with $5 \%$ milk at $20^{\circ} \mathrm{C}$ for $1 \mathrm{~h}$. Then it was incubated with the primary antibody (dilution, $1: 100$ ) at $4^{\circ} \mathrm{C}$ overnight. After sections were rinsed with tris-buffered saline with Tween-20 (TBST) 3 times, biotin-labeled secondary polyclonal antibody (cat. no. SA00004-11; Proteintech Group, Inc.) was added dropwise for incubation for $30 \mathrm{~min}$, and sections were rinsed again with TBST. Then the color was developed via diaminobenzidine (DAB) in the dark, and the development time was controlled, followed by re-staining using hematoxylin and sealing with gum.

Five fields of vision were randomly selected, and cells were graded and scored according to the percentage of positive cells and the staining intensity. No staining, light yellow, brown yellow and dark brown were denoted as $0,1,2$ and 3 points, respectively. The number of positive cells $<5 \%, 5-25 \%, 26-50 \%$ and $>50 \%$ were denoted as $0,1,2$ and 3 points, respectively. The two scores were added up, and the final score $\geq 3$ points indicated positive expression, while $<3$ points indicated negative expression.

Correlation analysis. According to the expression levels of p16 and nm23-H1 in STS tissues, 64 STS patients were 

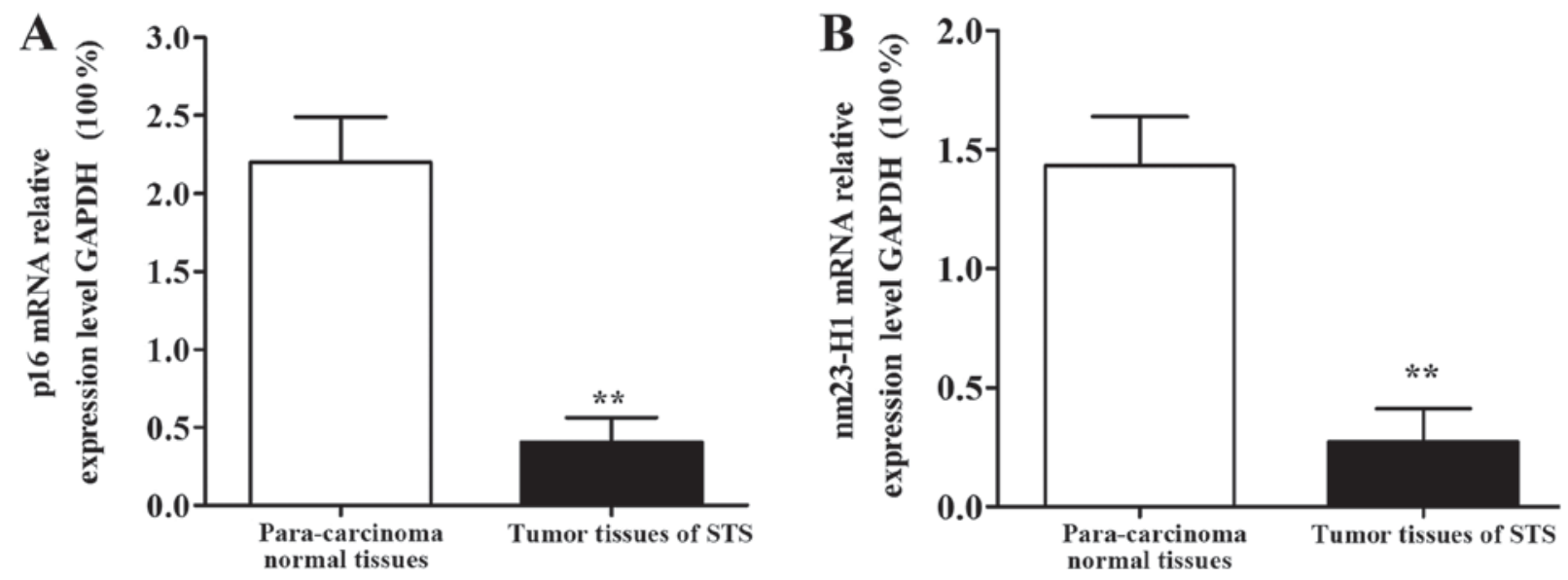

Figure 1. Detection of p16 and nm23-H1 mRNA expression levels in tissues of patients via RT-qPCR. p16 and nm23-H1 mRNA expression levels in tumor tissues of STS patients are significantly decreased compared with those in para-carcinoma normal tissues $\left({ }^{* *} \mathrm{P}<0.01\right)$. (A) p16 mRNA expression in tissues, (B) nm23-H1 mRNA expression in tissues. RT-qPCR, reverse transcription-quantitative polymerase chain reaction.
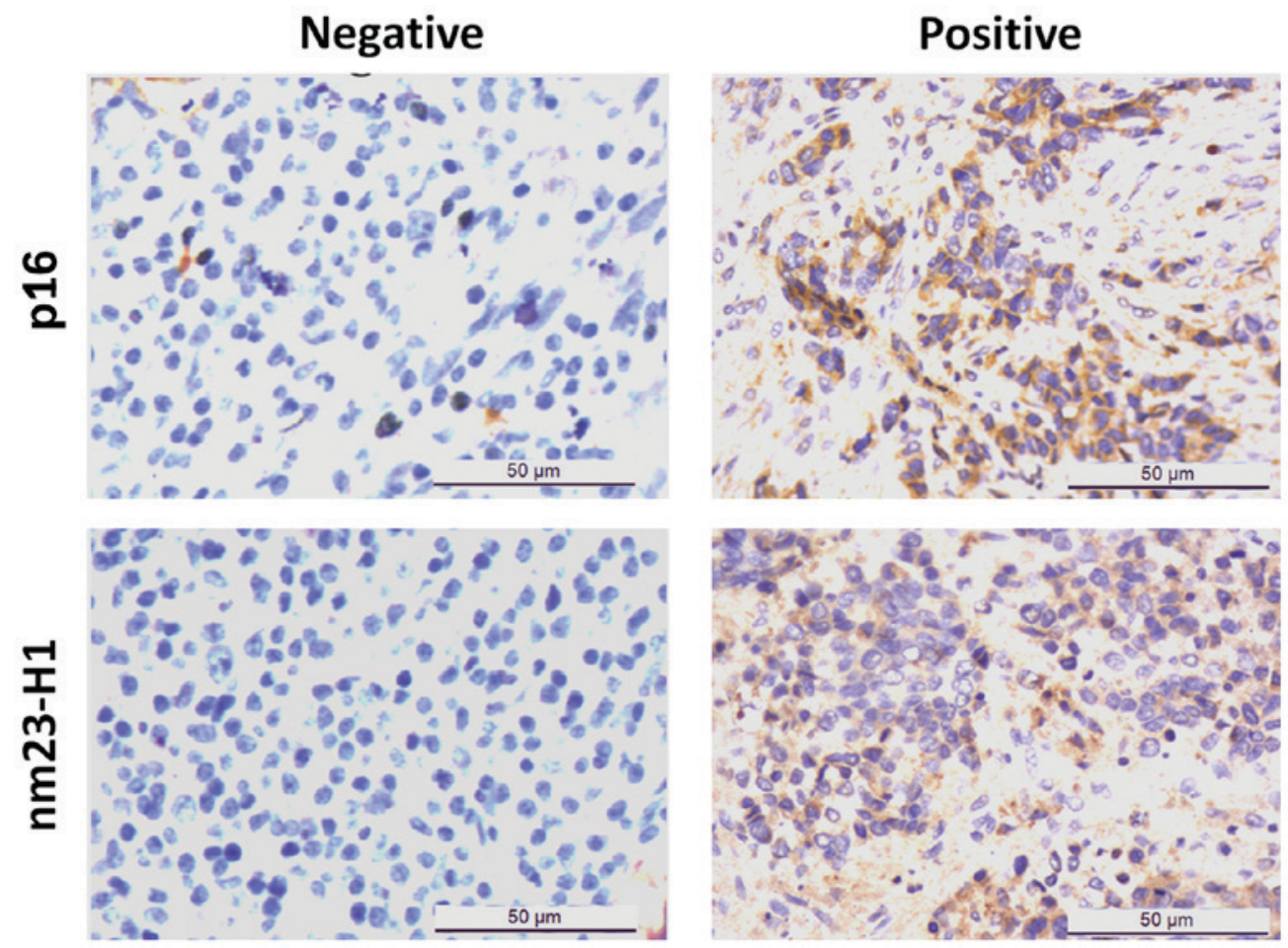

Figure 2. Detection of p16 and nm23-H1 protein expression levels in pathological tissues of patients via immunohistochemistry (magnification, $\mathrm{x} 400$ ).

divided into the p16-positive group, the p16-negative group, the nm23-H1-positive group and the nm23-H1-negative group. Spearman's correlation analysis was used to study the correlation between 16 and $\mathrm{nm} 23-\mathrm{H} 1 \mathrm{mRNA}$ expression and protein expression and the relationship between expression levels of p16 and nm23-H1 in STS tissues. Patients were followed up once a month from the first day after operation for a total of 5 years. Moreover, correlations of $\mathrm{p} 16$ and $\mathrm{nm} 23-\mathrm{H} 1$ expression levels with pathological parameters and prognosis of patients were analyzed combined with clinical and follow-up data.

Statistical analysis. Statistical Product and Service Solutions (SPSS) 17.0 (SPSS, Inc., Chicago, IL, USA) software was used for data processing. Measurement data were presented as mean \pm standard deviation, and t-test was used for the intergroup comparison. Chi-square test was used for the intergroup comparison of enumeration data, and Spearman's test was used for correlation analysis. Kaplan-Meier survival curves was used for survival curve analysis of STS patients with $\log$ rank test. $\mathrm{P} \leq 0.05$ was considered to indicate a statistically significant difference.

\section{Results}

p16 and nm23-H1 mRNA expression levels in tissue specimens. The results of RT-qPCR showed that p16 and nm23-H1 mRNA expression levels in tumor tissues of STS patients were significantly decreased compared with those in para-carcinoma 

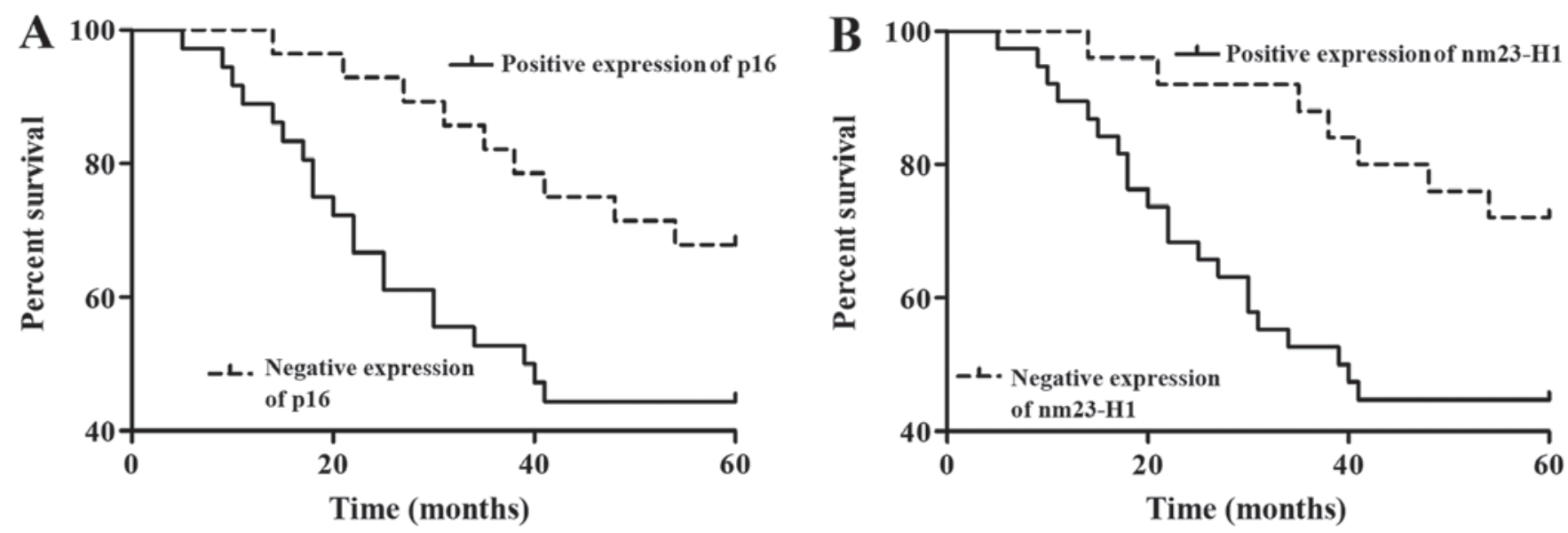

Figure 3. Correlations of p16 and nm23-H1 protein expression levels with survival of STS patients. (A) Kaplan-Meier survival curves of STS patients with positive and negative protein expression levels of p16. (B) Kaplan-Meier survival curves of STS patients with positive and negative protein expression levels of nm23-H1.

Table III. Correlation of abnormal expression levels of p16 and nm23-H1 with pathological parameters of STS patients.

\begin{tabular}{|c|c|c|c|c|c|c|c|}
\hline \multirow[b]{2}{*}{$\begin{array}{l}\text { Pathological } \\
\text { parameters }\end{array}$} & \multirow[b]{2}{*}{$\mathrm{n}$} & \multicolumn{3}{|c|}{ p16 $(n=36)$} & \multicolumn{3}{|c|}{ nm23-H1 (n=39) } \\
\hline & & $\begin{array}{l}\text { Negative } \\
{[\mathrm{n}(\%)]}\end{array}$ & $\chi^{2}$ value & P-value & $\begin{array}{c}\text { Negative } \\
{[\mathrm{n}(\%)]}\end{array}$ & $\chi^{2}$ value & P-value \\
\hline Sex & & & 0.12 & $>0.05$ & & 0.74 & $>0.05$ \\
\hline Male & 35 & $19(54.29)$ & & & $23(65.71)$ & & \\
\hline Female & 29 & $17(58.62)$ & & & $16(55.17)$ & & \\
\hline Age (years) & & & 0.70 & $>0.05$ & & 0.19 & $>0.05$ \\
\hline$\geq 60$ & 26 & $13(50.00)$ & & & 15 (57.69) & & \\
\hline$<60$ & 38 & $23(60.52)$ & & & $24(63.16)$ & & \\
\hline Tumor size (cm) & & & 9.80 & $<0.01$ & & 0.42 & $>0.05$ \\
\hline$\geq 5$ & 39 & $28(71.79)$ & & & $25(64.10)$ & & \\
\hline$<5$ & 25 & $8(32.00)$ & & & $14(56.00)$ & & \\
\hline Tumor metastasis & & & 7.52 & $<0.01$ & & 9.12 & $<0.01$ \\
\hline Yes & 33 & $25(75.76)$ & & & $26(78.79)$ & & \\
\hline No & 31 & $12(38.71)$ & & & 13 (41.94) & & \\
\hline Clinical staging & & & 8.13 & $<0.01$ & & 8.50 & $<0.01$ \\
\hline I & 22 & $7(31.82)$ & & & 8 (36.36) & & \\
\hline II-III & 42 & $29(69.05)$ & & & $31(73.81)$ & & \\
\hline
\end{tabular}

normal tissues, and differences were statistically significant $(\mathrm{P}<0.01)$ (Fig. 1).

p16 and nm23-H1 protein expression levels in STS tissues. Results of immunohistochemical detection showed that the positive immunohistochemical staining of p16 and nm23-H1 was dark brown, and p16 staining was mainly located in the nucleus and cytoplasm. nm23-H1 staining was mainly located in the cytoplasm (Fig. 2). Immunohistochemical scores showed that the positive expression rate of p16 in STS tissues was $43.75 \%$ (28/64), which was significantly lower than that in para-carcinoma normal tissues $(85.93 \%, 55 / 64)$. The positive expression rate of nm23-H1 in STS tissues was 39.06\% (25/64), which was also significantly lower than that in para-carcinoma normal tissues $(89.06 \%, 57 / 64)$. Differences were statistically significant $(\mathrm{P}<0.01)$.

Correlation between p16 and nm23-H1 mRNA expression and protein expression. Correlation between the expression of p16 and nm23-H1 mRNA and the expression of protein was analyzed by Spearman's test. The results showed that the expression of p16 and $\mathrm{nm} 23-\mathrm{H} 1 \mathrm{mRNA}$ was positively correlated with the expression of protein ( $\mathrm{p} 16: \mathrm{r}=0.92, \mathrm{P}<0.01$; $\mathrm{nm} 23-\mathrm{H} 1: \mathrm{r}=0.95, \mathrm{P}<0.01)$.

Correlation between 116 and nm23-H1 protein expression levels in STS tissues. Among the 64 cases of STS tissues, p16 and nm23-H1 proteins were positive in 20 cases, p16 and 
$\mathrm{nm} 23-\mathrm{H} 1$ proteins were negative in 31 cases, $\mathrm{p} 16$ protein was positive and $\mathrm{nm} 23-\mathrm{H} 1$ protein was negative in 8 cases, $\mathrm{p} 16$ protein was negative and $\mathrm{nm} 23-\mathrm{H} 1$ protein was positive in 5 cases. Results of Spearman's correlation analyses showed that there was a positive correlation between the expression levels of p16 and nm23-H1 proteins (correlation coefficient $\mathrm{r}=0.722)$, and the correlation had statistical significance $(\mathrm{P}<0.01)$ (Table II).

Correlation of p16 and nm23-H1 expression levels with pathological parameters of STS patients. Analysis results of the relationship of pathological parameters of STS patients with p16 and nm23-H1 expression levels are shown in Table III. Chi-square test showed that the negative expression of p16 was correlated with tumor size, metastasis and staging in patients $(\mathrm{P}<0.01)$, but had no correlation with sex and age of patients $(\mathrm{P}>0.05)$. Besides, the negative expression of $\mathrm{nm} 23-\mathrm{H} 1$ was correlated with tumor metastasis and staging in patients $(\mathrm{P}<0.01)$, but had no correlations with sex, age and tumor size $(\mathrm{P}>0.05)$.

Correlation of p16 and nm23-H1 protein expression levels with survival of STS patients. Results of the 5-year follow-up revealed that of the 64 patients, 35 cases survived and 29 cases had died, and the overall 5-year survival rate was $54.68 \%$. Kaplan-Meier survival curves was used for survival curve analysis of STS patients with log rank test. Patients with negative protein expression levels of p16 and nm23-H1 had poorer survival. Univariate survival analyses showed that both p16 and nm23-H1 could significantly affect the overall survival rate of STS patients $(\mathrm{P}<0.05$; Fig. 3$)$.

\section{Discussion}

Soft tissue sarcoma (STS), as a kind of malignant tumor derived from mesoderm, has higher heterogeneity and many different subtypes (12). Although its incidence rate is not high, STS has higher recurrence and metastasis rates. Clinical statistics have shown that the overall 3-year and 5-year survival rates of STS patients were 77 and $75 \%$, the recurrence rates were 28 and $37 \%$, and the metastasis rates were 35 and $43 \%$, respectively (13). Studies have shown that the positive margin is the most important factor affecting the recurrence and metastasis of STS $(14,15)$.

P16 gene, a CDK inhibitor, encodes the p16 protein that could specifically bind to CDK4 or CDK6 and competitively affect the binding of cyclin $\mathrm{D}$, thereby inhibiting the activity of CDK4 and CDK6, leading to cell cycle arrest, and inhibiting tumor cell growth $(16,17)$. Studies have proven that there are gene deletion, mutation, methylation and other abnormalities in p16 in a variety of tumor cell lines and solid tumors, so it is speculated that p16 gene plays a key role in tumor formation and development processes (18).

$\mathrm{Nm} 23$ gene is located on human chromosome 17q22, which encodes a protein containing 152 amino acids and having a high homology to the amino acid sequence of nucleoside diphosphate kinase (NDPK) $(19,20)$. Therefore, it is speculated that nm23 gene may exert a similar effect to NDPK, and that it can play an important role in regulating cell signal transduction and cell differentiation. There are 2 subtypes of human nm23 gene, namely nm23-H1 and nm23-H12, the former of which plays an important role in inhibiting tumor metastasis (21). It was found that $\mathrm{nm} 23-\mathrm{H} 1$ can inhibit the metastasis of a variety of malignant tumors, and its expression level is negatively correlated with tumor invasion and metastasis. Moreover, tumor patients with high $\mathrm{nm} 23-\mathrm{H} 1$ expression have better prognosis (8).

In order to further investigate the expression levels of p16 and nm23-H1 in tumor tissues of STS patients and their influence on the pathological parameters and prognosis of STS patients, RT-qPCR and immunohistochemical assay were performed in this study. Detection results showed that the expression levels of p16 and nm23-H1 in tumor tissues of STS patients were significantly lower than those in para-carcinoma normal tissues $(\mathrm{P}<0.01)$. Results of immunohistochemical assay showed that the positive expression rates of p16 and nm23-H1 in tumor tissues of STS patients (43.75 and $39.06 \%$, respectively) were significantly lower than those in para-carcinoma normal tissues (85.93 and 89.06\%, respectively). The results of Spearman's test showed that the expression of p16 and nm23-H1 mRNA was positively correlated with the expression of protein. p16 is a CDK4 inhibitor protein, and some scholars have proposed that nm23 may regulate the function of p53 gene at CDK level. Correlation analyses in this study showed that there was a significantly positive correlation between the expression levels of p16 and $\mathrm{nm} 23-\mathrm{H} 1$, indicating that p16 and nm 23 genes may have a close correlation in STS, and its specific molecular process and mechanism need to be studied more profoundly. Along with clinicopathological parameters and the survival statistical analysis of patients, it was found that the negative expression of p16 was correlated with tumor size, metastasis and staging in patients, but had no correlation with the sex and age of patients. Besides, the negative expression of nm23-H1 was correlated with tumor metastasis and staging in patients, but had no correlation with sex, age and tumor size in patients. The 64 STS patients were followed up for 5 years, and results showed that the overall 5-year survival rate of patients was $54.68 \%$, and patients with positive expression levels of p16 and nm23-H1 had a better prognosis. Moreover, univariate survival analyses showed that both p16 and nm23-H1 could affect the overall survival rate of STS patients.

In conclusion, the expression of p16 and nm23-H1 is low in STS, and their expression levels are closely related to the pathological parameters and prognosis of STS patients, so they can serve as reference indexes for prognosis estimation of STS.

\section{Acknowledgements}

Not applicable.

\section{Funding}

No funding was received.

\section{Availability of data and materials}

All data generated or analyzed during this study are included in this published article. 


\section{Authors' contributions}

JJ performed the data analyses and wrote the manuscript. $\mathrm{PY}$ and XZha contributed significantly to data analysis and manuscript preparation. FY performed RT-qPCR. GS and WB helped with data analysis. GH and MX collected the data and were responsible for statistical analysis. WW was a major contributor in performing immunohistochemical assay. XZhe performed the data analysis with constructive discussions. All authors read and approved the final manuscript.

\section{Ethics approval and consent to participate}

This study was reviewed and approved by the Clinical Ethics Committee of General Hospital of Chinese People's Liberation Army (Beijing, China) and all patients or their families signed an informed consent.

\section{Patient consent for publication}

Not applicable.

\section{Competing interests}

The authors declare that they have no competing interests.

\section{References}

1. Doyle LA: Sarcoma classification: An update based on the 2013 World Health Organization Classification of Tumors of Soft Tissue and Bone. Cancer 120: 1763-1774, 2014

2. Fletcher CDM, Bridge JA, Hogendoorn PCW and Mertens F (eds): WHO Classification of Tumours of Soft Tissue and Bone. Fourth Edition. Vol. 5. IARC Press, Lyon, 95-104, 2013.

3. Jemal A, Bray F, Center MM, Ferlay J, Ward E and Forman D: Global cancer statistics. CA Cancer J Clin 61: 69-90, 2011.

4. Shi YQ, Zong XY, Wang J and Li S: Clinical analysis of 251 cases of soft tissue sarcomas. Zhonghua Wai Ke Za Zhi 41: 116-118, 2003 (In Chinese).

5. Bannasch H, Eisenhardt SU, Grosu AL, Heinz J, Momeni A and Stark GB: The diagnosis and treatment of soft tissue sarcomas of the limbs. Dtsch Arztebl Int 108: 32-38, 2011.

6. Ruymann FB and Grovas AC: Progress in the diagnosis and treatment of rhabdomyosarcoma and related soft tissue sarcomas. Cancer Invest 18: 223-241, 2000.

7. Kashiwabara K, Oyama T, Sano T, Fukuda T and Nakajima T: Correlation between methylation status of the p16/CDKN2 gene and the expression of p16 and Rb proteins in primary non-small cell lung cancers. Int J Cancer 79: 215-220, 1998.
8. Steeg PS, Bevilacqua G, Kopper L, Thorgeirsson UP, Talmadge JE, Liotta LA and Sobel ME: Evidence for a novel gene associated with low tumor metastatic potential. J Natl Cancer Inst 80: 200-204, 1988.

9. Parhar RS, Shi Y, Zou M, Farid NR, Ernst P and al-Sedairy ST: Effects of cytokine-mediated modulation of $n m 23$ expression on the invasion and metastatic behavior of B16F10 melanoma cells. Int J Cancer 60: 204-210, 1995.

10. Okada K, Urano T, Goi T, Baba H, Yamaguchi A, Furukawa K, Shiku H: Isolation of human nm23 genomes and analysis of loss of heterozygosity in primary colorectal carcinoma using a specific genomic probe. Cancer Res 54: 3979-3982, 1994.

11. Livak KJ and Schmittgen TD: Analysis of relative gene expression data using real time quantitative PCR and the 2( Delta Delta C(T)) method. Methods 25: 402 408, 2001.

12. Jemal A, Siegel R, Ward E, Hao Y, Xu J and Thun MJ: Cancer statistics, 2009. CA Cancer J Clin 59: 225-249, 2009.

13. Casali PG and Blay JY; ESMO/CONTICANET/EUROBONET Consensus Panel of experts: Soft tissue sarcomas: ESMO Clinical Practice Guidelines for diagnosis, treatment and follow-up. Ann Oncol 21 (Suppl 5): v198-v203, 2010.

14. Campos M, De Campos SG, Ribeiro GG, Eguchi FC, Silva SR, De Oliveira CZ, Da Costa AM, Curcelli EC, Nunes MC, Penna V, et al: Ki-67 and CD100 immunohistochemical expression is associated with local recurrence and poor prognosis in soft tissue sarcomas, respectively. Oncol Lett 5: 1527-1535, 2013.

15. Ch'ng E, Tomita Y, Zhang B, He J, Hoshida Y, Qiu Y, Morii E, Nakamichi I, Hamada K, Ueda T, et al: Prognostic significance of CD100 expression in soft tissue sarcoma. Cancer 110: 164-172, 2007.

16. Merlo A, Herman JG, Mao L, Lee DJ, Gabrielson E, Burger PC, Baylin SB and Sidransky D: 5' CpG island methylation is associated with transcriptional silencing of the tumour suppressor p16/CDKN2/MTS1 in human cancers. Nat Med 1: 686-692, 1995.

17. Reimers N, Kasper HU, Weissenborn SJ, Stützer H, Preuss SF, Hoffmann TK, Speel EJ, Dienes HP, Pfister HJ, GuntinasLichius O, et al: Combined analysis of HPV-DNA, p16 and EGFR expression to predict prognosis in oropharyngeal cancer. Int J Cancer 120: 1731-1738, 2007.

18. Fan X, Yu K, Wu J, Shao J, Zhu L and Zhang J: Correlation between squamous cell carcinoma of the lung and human papillomavirus infection and the relationship to expression of p53 and p16. Tumour Biol 36: 3043-3049, 2015.

19. Postel EH: NM23-NDP kinase. Int J Biochem Cell Biol 30: 1291-1295, 1998.

20. Valentijn LJ, Koster J and Versteeg R: Read-through transcript from NM23-H1 into the neighboring NM23-H2 gene encodes a novel protein, NM23-LV. Genomics 87: 483-489, 2006.

21. Lacombe ML, Milon L, Munier A, Mehus JG and Lambeth DO: The human nm23/nucleoside diphosphate kinases. J Bioenerg Biomembr 32: 247-258, 2000.

This work is licensed under a Creative Commons Attribution-NonCommercial-NoDerivatives 4.0 International (CC BY-NC-ND 4.0) License. 